\title{
Concentration of blood lead and ethnicity in the United Kingdom
}

\author{
S T Kolev, I House, G Bell, D Shaw, V Murray
}

\begin{abstract}
The relation between concentration of blood lead and ethnic background in 779 children was examined with the analytical results from the trace element service at the Medical Toxicology Unit (MTU), Guy's and St Thomas's Hospital Trust for the period 1980-94. The ethnic identity was determined with the first and the second names of the investigated subjects. Of the patients of European origin (European) studied $72 \cdot 8 \% v$ only $50 \cdot 6 \%$ of the children with origins in the Indian subcontinent (Asian) had a concentration of blood lead $<100 \mu \mathrm{g} / 1$. The percentage of subgroups with concentrations above the upper acceptable limit of $200 \mu \mathrm{g} / \mathrm{l}$ was significantly higher in Asian subjects (European 5\% $v$ Asian 26.5\%), with the most pronounced difference in those with concentrations of blood lead of $500 \mu \mathrm{g} / 1$ (European 0.8\% v Asian 10.5\%). This study shows that a correlation exists between Asian ethnic background and concentration of blood lead in children. Factors such as cultural habits-for example, use of traditional remedies, cosmetics, diet-and socioeconomic status, may have contributed to this result.
\end{abstract}

(Occup Environ Med 1996;53:841-843)

Keywords: blood lead, ethnicity

Lead poisoning continues to be a common environmentally induced disease in the paediatric population worldwide. The adverse effects of lead are seen primarily in the developing brain and the nervous system. Children seem to be more susceptible than adults to the toxic effects of lead because of incomplete development of the blood brain barrier, greater intestinal absorption of lead, and a tendency to put objects or their hands into their mouths (pica habit) thereby increasing ingestion of contaminated substances. ${ }^{1}$ Also, they have a smaller proportion of dense bone tissue than adults and this prevents the transfer of the absorbed lead into bone, and it remains in the soft tissues where it produces toxic effects. ${ }^{2}$ Childhood lead poisoning has been docu- mented in all socioeconomic groups, geographical areas, and racial and ethnic groups. ${ }^{3}$ The possibility that the Asian community in the United Kingdom has a high exposure to lead due to the use of traditional remedies and cosmetics containing lead has been suggested in numerous reports. ${ }^{45}$ Several well documented cases of lead intoxication associated with these products indicate that their use may significantly contribute to the overall incidence of lead toxicity in the Asian population. However, the relative risk in this ethnic group has not been clearly established.

The Trace Element Laboratory of the Medical Toxicology Unit at Guy's and St Thomas's Hospital Trust provides a comprehensive range of analytical services together with relevant medical toxicology assessment to a wide geographical area. In this study we examined the analytical results from the trace element service for the period 1980-94, and investigated whether a correlation existed between the ethnic background and increased concentration of blood lead.

\section{Method}

Of a total of 8400 blood lead measurements, 1325 were on samples known to be from children ( $<10$ years old). Samples were collected by venepuncture. All samples were analysed by graphite furnace atomic absorption spectrometry after dilution. Accuracy was $1 \%$ of true volume, relative SD was $2 \%-3 \%$ in the short term at $200 \mathrm{~g} / 1$. Three internal quality control materials were analysed at the start and end of every batch. If there were more than 10 samples in a batch, all three internal quality control materials were analysed after every 10th sample. A blank was analysed at this point as well as at the start and end of every batch. The Medical Toxicology Unit laboratory participates in two quality assurance schemes for concentration of blood lead. The score is good to excellent in both schemes.

Typical reasons for blood lead assay being requested were essentially the same for both ethnic groups and included anaemia, developmental delay, pica habit, chronic abdominal pain, and behavioural problems. Only the first result for each child was used, giving 905 results for analysis. As ethnic origin was not 
routinely recorded, the first and the last names were used to determine the ethnic identity of the investigated subjects. The sensitivity, specificity, and predictive values of this method have been ascertained, and were found to have high reliability, particularly if both first and second names were used." Any subjects with names that were unclear were excluded from further analysis as were those subjects with African, Chinese, or Arab names. This left 779 children who had blood lead measured and were either European or had ethnic origin in the Indian subcontinent. All studied subjects were divided into subgroups by the concentration of blood lead. Frequency distributions were calculated for sex, age, and concentration of blood lead, and the $\chi^{2}$ statistic was calculated for each of these, between those whose ethnic origin was European and those whose ethnic origin was from the Indian subcontinent. The frequencies were then expressed as the percentage of the total number of cases in each ethnic group.

\section{Results}

The $\chi^{2}$ test between concentration of blood lead and ethnic groups showed a significant difference $\left(\chi^{2}=86.83\right.$, degrees of freedom (df) $5, \mathrm{P}<0.0001$, figure). Of the European patients studied $72 \cdot 8 \%$ had a blood lead concentration $<100 \mu \mathrm{g} / 1$ compared with only $50.6 \%$ of the Asian children. The percentage of European subjects with increased concentrations of blood lead tended to decline as the concentration of blood lead increased. The percentage of children with concentrations of $100-200 \mu \mathrm{g} / 1$ was similar in both ethnic groups. However, in subgroups with concentrations above the upper acceptable limit of $200 \mu \mathrm{g} / \mathrm{l}$, the percentage was significantly higher in Asian subjects (European 30/599, Asian 48/180, $\mathrm{P}<0.0001$ ) This difference was even more pronounced in cases with concentrations $>600 \mu \mathrm{g} / \mathrm{l}$ (European 0.5\%; Asian $7 \cdot 2 \%$, data not shown).

No significant sex and age differences were found between the European and Asian ethnic groups. It was noted, however, that many more boys had had their blood lead measured than girls in both groups (European: male $61 \%$, female $37 \%$, unknown $2 \%$; Asian: male $48 \%$, female $36 \%$, unknown $16 \%$ ).

\section{Discussion}

The results from this study show that a small

Distributions of blood lead concentrations and ethnicity. The results are expressed as the percentage of the total number of cases in each ethnic group.

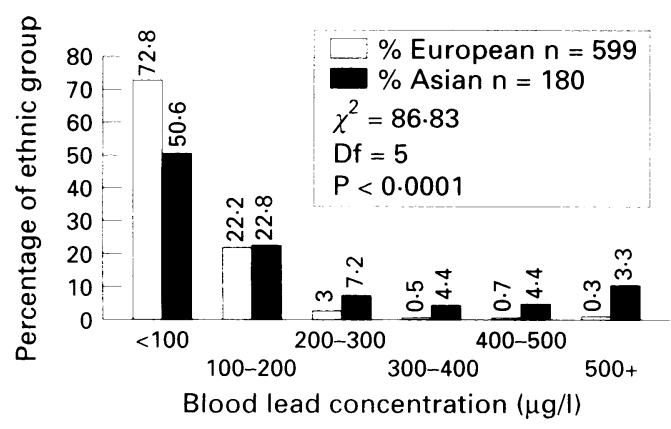

but important number of children have been exposed to lead concentrations that could result in lead toxicity. The incidence of lead concentrations above the upper acceptable limit of $200 \mu \mathrm{g} / \mathrm{l}$ was significantly higher in Asian subjects. The present findings are consistent with earlier surveys carried out on preschool children, but they are at variance with a more recent study of blood lead concentration in children living in the United Kingdom, which did not find a correlation between increased concentration of blood lead and ethnic origin.

Decreased activity of the enzyme $\delta$ aminolevolinic acid dehydratase (ALAD) is the earliest biochemical effect on the synthesis of haemoglobin once lead concentrations are $>50 \mu \mathrm{g} / \mathrm{l}$. The abnormal protoporphyrin (ZPP) production starts to increase at blood concentrations of $200-300 \mu \mathrm{g} / \mathrm{l}$. With increasing lead exposure, interference in the production of haemoglobin leads to anaemia. A decrease in the conductivity of the peripheral nervous system is usually found at a concentration $>500 \mu \mathrm{g} / \mathrm{l}$. The central nervous system of children is particularly sensitive to the negative effects from lead exposure and adverse effects have been clearly documented for blood lead concentrations of $300 \mu \mathrm{g} / \mathrm{l}$."

The primary reservoirs for lead exposure in children are lead based paints in older housing, soil, air, water, and dust contaminated from industrial sources and vehicle emissions. Exposure to traditional remedies and cosmetics containing lead has also been identified as a potential cause of childhood toxicity in the Asian community in the United Kingdom. Several of these products obtained in the Indian subcontinent and in Britain were found to contain high concentrations of heavy metals. Surma samples have been found to contain up to $86 \%$ lead. ' A correlation between the use of these products and increased blood lead concentrations has been shown' Also, modes of absorption of the products have been examined as well as the implications for mental health." The higher rates of increased concentration of blood lead among the Asian children throughout the investigated period suggest that, despite the educational campaign and the government health warnings in the past, lead poisoning from Asian traditional remedies and cosmetics still remains a cause of concern.

Other determinants such as socioeconomic and cultural factors including children's habits-for example, pica habit-might be involved. The importance of geographical location and the socioeconomic status in the aetiology of childhood lead poisoning has been emphasised in a recent publication.' The results of our study reflect an overall occurrence of significantly increased concentrations of blood lead in Asian children without reference to these factors. The higher number of Asian children residing in urban areas and differences in their economic status may, therefore, have contributed to our results.

The significant difference in the concentration of blood lead in the two groups studied indicates the need for a different approach to 
the screening and management of these children. In a 13 month study in California there were 40 cases with blood lead concentrations $>200 \mu \mathrm{g} / 1$ in children who had been treated with traditional remedies and cosmetics. Of particular concern was the finding that $60 \%$ of these children were asymptomatic and this included five cases who had blood lead concentrations $>500 \mu \mathrm{g} / 1 .^{1}$ In the present study the concentrations of blood lead were only analysed for symptomatic patients. As children with increased concentration of blood lead do not always develop features of intoxication, it is possible that the results represent only a small proportion of those cases. Failure to thrive and anaemia were the most common reasons for blood lead assay being requested. Furthermore, higher prevalence of iron deficiency has been reported in Asian children, (39\% in Asian children and $16 \%$ in white children). A haemoglobin concentration of $110 \mathrm{~g} / 1$ was considered to indicate anaemia. This study population comprised children aged 15 to 24 months. ${ }^{11}$ Therefore, the concentration of blood lead in Asian children is more likely to be investigated than in European children and this may have resulted in better detection of otherwise asymptomatic cases in this group.

This study shows that a correlation exists between Asian ethnic background and concentration of blood lead in children. Further studies will be necessary to find the precise role of the use of traditional remedies and cosmetics and their importance as a determinant of lead poisoning in this ethnic group, and whether additional social and economic factors are involved. We think that emphasis on public education programmes together with monitoring of the populations at risk - that is, young children-is needed to identify the sources of lead exposure and effectively reduce the number of cases of clinical lead poisoning in children.

We thank Dr Sukhdev S Sharma (seconded to Medical Toxicology Unit), Dr Susan Schonfield, and Heather Wiseman for their assistance.

1 Knodel LC. Lead: continuing environmental threat to children. Toxic Substance Mechanisms 1995;14:61-3.

2 Al-Salen I, Devol E, Taylor A. Distribution of blood lead levels in 1047 Saudi Arabian children with respect to province, sex, and age. Arch Environ Health 1994;49: province,

3 Rifai N, Cohen G, Wolf M, Cohen L, Faser C, Savory J DePalma L. Incidence of lead poisoning in young children from inner-city, suburban, and rural communities Ther Drug Monit 1993;15:71-4.

4 Ali AR, Smales ORC, Aslam M. Surma and lead poisoning. $B M \mathcal{F} 1978 ; 2: 915-6$

5 Bayly GR, Braithwaite RA, Sheehan TMT, Dyer NH, Grimley C, Ferner RE. Lead poisoning from Asian traditional remedies in the West Midlands-report of a series of five cases. Hum Exp Toxicol 1995;14:24-8.

6 Nicoll A, Bassett K, Ulijaszek S. What's in a name? $f$ Epidemiol Community Health 1986;40:364-8.

7 Department of the Environment Central Directorate on Environmental Pollution. Blood lead concentration in preschool children in Birmingham. London: HMSO, 1982. school children in Birming
(Pollution report No 15.)

8 Singal GM, Gatrad AR, Howse PM, Johnson KW, Ganley $\mathrm{M}$, Thomas A, et al. Blood lead, ethnic origin, and lead exposure. Arch Dis Child 1988;63:973-5.

9 Elinder CG, Friberg L, Kjellstrom T, Nordberg G, Oberdoerster $\mathrm{G}$, eds. Inorganic lead. In: Biological monitoring of metals. Geneva: Investigative Programme of Chemical Substances, World Health Organisation, 1994: 54-8.

10 Fernando NP, Healy MA, Aslam M, Davis SS, Hussein A Lead poisoning and traditional practices: the consequences for world health. A study in Kuwait. Public Health London 1981;95:250-60.

11 Marder E, Nicoll A, Polnay L, Shulman CE. Discovering anaemia at child health clinics. Arch Dis Child 1990;65 892-4. 